\title{
Retraction Note to: Single-nucleotide polymorphisms of LIG1 associated with risk of lung cancer
}

\author{
Yan-Zhi Chen $^{1} \cdot$ Zeng-Hui Fan ${ }^{2} \cdot$ Yu-Xia Zhao ${ }^{1} \cdot$ Lu Bai $^{3} \cdot$ Bao-Shen Zhou ${ }^{4}$. \\ Hai-Bo Zhang ${ }^{5}$ - Dan Liu
}

Published online: 17 August 2015

(C) International Society of Oncology and BioMarkers (ISOBM) 2015

Retraction to: Tumor Biol. (2014) 35:9229-9232

DOI 10.1007/s13277-014-2199-Z

The Publisher and Editor retract this article in accordance with the recommendations of the Committee on Publication Ethics (COPE). After a thorough investigation we have strong reason to believe that the peer review process was compromised.

The online version of the original article can be found at http://dx.doi.org/ 10.1007/s13277-014-2199-z.

Yu-Xia Zhao

cmu4h_zyx@yeah.net

1 Department of Radiation Oncology, The Fourth Affiliated Hospital of China Medical University, Chongshan East Street No. 4, Yuhong District, Shenyang 110032, People's Republic of China

2 Department of Radiation Oncology, Chinese Medicine Hospital in Linyi City, Jiefang Street No. 211, Lanshan District, Linyi 276000 , People's Republic of China

3 Department of Radiation Oncology, The First Affiliated Hospital of China Medical University, 110001, Shenyang 110032, People's Republic of China

4 Department of Radiation Oncology, The General Hospital of Shenyang Military Region, Shenyang 110032, People's Republic of China

5 Department of Epidemiology, School of Public Health, China Medical University, Shenyang 110032, People's Republic of China 